\title{
Modelo de Oposições Múltiplas Modificado: abordagem baseada em traços distintivos
}

\author{
Modified Multiple Oppositions' Model: approach based on distinctive \\ features
}

\author{
Karina Carlesso Pagliarin¹, Marizete Ilha Ceron², Márcia Keske-Soares ${ }^{3}$
}

\begin{abstract}
RESUMO
O objetivo deste trabalho foi propor uma abordagem com enfoque em traços distintivos para o Modelo de Oposições Múltiplas e testar sua aplicação em um sujeito falante do Português Brasileiro. O Modelo foi aplicado em um sujeito do sexo masculino, com desvio fonológico moderado-severo e idade de seis anos e meio. Após avaliação fonológica, procedeu-se à seleção dos sons-alvo. Considerouse o sistema fonológico inicial, bem como os traços distintivos alterados ([+voz], [+soante], [+aproximante], [coronal/ \pm anterior], [-contínuo]). Os traços com maior número de alterações eram [+voz, +soante, +aproximante], justificando a escolha dos seguintes sons-alvo para tratamento: /r/ x /1/ x /K/ x /3/ x /z/, em onset medial. Tendo como base a aplicação desse modelo em falantes do inglês, percebeu-se a necessidade de introduzir alguns procedimentos, como: a escolha dos sons-alvo a partir dos traços distintivos alterados e a estrutura da sessão terapêutica. Ao final, o sujeito foi reavaliado a fim de verificar as aquisições no sistema fonológico. Após dez sessões de fonoterapia, o sujeito adquiriu os fonemas $/ 1, K, 3, \mathrm{z} /$, generalizando-os para outras posições na palavra $(/ / 3 / \mathrm{e} / \mathrm{l} /$ em onset inicial). Verificou-se, também, a ocorrência de generalização para a mesma classe e para outras classes de sons (/b/, /d/, /g/, /v/). No entanto, o fonema /r/ não foi adquirido. Esta proposta mostrou-se efetiva, pois o sujeito apresentou melhora em seu sistema fonológico com poucas sessões terapêuticas. Pôde-se observar que o Modelo de Oposições Múltiplas, utilizando a abordagem com base em traços distintivos é eficaz, pois favoreceu diversas mudanças fonológicas.
\end{abstract}

Descritores: Distúrbio fonológico; Fala; Fonoterapia; Generalização; Criança; Relatos de casos

\section{INTRODUÇÃO}

Existem vários modelos de terapia baseados na fonologia, para o tratamento dos desvios fonológicos (DF), os quais se diferenciam segundo a teoria fonológica que os fundamenta, os princípios terapêuticos e o modo como são aplicados. Dentre os mais utilizados e aplicados, principalmente no sul do Brasil, estão: Ciclos Modificado ${ }^{(1)}$, Modelo de Pares Mínimos/ Oposições Máximas ${ }^{(2)}$, Oposições Máximas Modificado ${ }^{(3)}$, e o

Trabalho realizado na Universidade Federal de Santa Maria - UFSM - Santa Maria (RS), Brasil.

(1) Pós-graduanda (Mestrado) em Distúrbios da Comunicação Humana pela Universidade Federal de Santa Maria - UFSM - Santa Maria (RS), Brasil; Professora do Curso de Fonoaudiologia da Universidade Federal de Santa Maria - UFSM - Santa Maria (RS), Brasil.

(2) Pós-graduanda (Mestrado) em Distúrbios da Comunicação Humana pela Universidade Federal de Santa Maria - UFSM - Santa Maria (RS), Brasil; Bolsista CAPES.

(3) Doutora, Professora do Curso de Fonoaudiologia e do Mestrado em Distúrbios da Comunicação Humana da Universidade Federal de Santa Maria - UFSM - Santa Maria (RS), Brasil.

Endereço para correspondência: Karina Carlesso Pagliarin. R. Coronel Scherer, 09, Centro, São Pedro do Sul (RS), Brasil, CEP: 97400-000. E-mail: karinap_fono@yahoo.com.br

Recebido em: 2/7/2008; Aceito em: 14/12/2008
Modelo "ABAB - Retirada e Provas Múltiplas"(4). O Modelo de Oposições Múltiplas ${ }^{(5)}$ trata-se de um modelo que pressupõe a abordagem contrastiva, porém não foi, ainda, aplicado em crianças falantes do português.

O Modelo de Oposições Múltiplas ${ }^{(5)}$ foi proposto como um modelo alternativo utilizado no tratamento de crianças com DF severos falantes do inglês. Essas crianças, em geral, substituem diversos fonemas do sistema adulto por um único som. Assim, a função contrastiva de muitos sons está ausente, o que reduz muito a inteligibilidade de fala e produz muitos homônimos. Esse modelo baseia-se na premissa de que o nível sistêmico da organização fonológica é essencial na descrição e intervenção dos sistemas de sons desviantes.

Nesse modelo, a criança é confrontada com vários sons simultaneamente, considerando-se, também, o fonema substituído. Além disso, o modelo é único para cada criança, pois cada uma possui suas próprias estratégias ou modelos fonológicos a serem corrigidos ${ }^{(5)}$.

O Modelo de Oposições Múltiplas é uma variação do modelo de pares mínimos/oposições máximas e é designado especificamente para o tratamento dos múltiplos fonemas substituídos pela criança com relação ao sistema adulto. $\mathrm{O}$ tratamento promove um ajustamento sistemático de um gran- 
de grupo contrastivo de sons, não apenas uma série de pares $\operatorname{mínimos}^{(6)}$.

Ao contrário do modelo de pares mínimos/oposições máximas, o modelo de oposições múltiplas não leva em consideração a ordem cronológica em que os sons são adquiridos (anterior ou posterior), o conhecimento que a criança possui do som ou se é ou não-estimulável. A seleção do alvo é baseada em dois parâmetros: classificação máxima e distinção máxima. A classificação máxima envolve a seleção de alvos que apresentam diferente ponto, modo e sonoridade dentro do sistema adulto, sendo o alvo selecionado a partir do som substituído. Enquanto que na distinção máxima, os alvos e o fonema substituído apresentam distinção máxima de $\operatorname{traços}^{(7)}$.

Tendo como base a aplicação desse modelo em falantes do inglês, percebeu-se a necessidade de avaliar a introdução de alguns procedimentos, como estimulação auditiva, trabalho com os pais e, principalmente, a abordagem teórica para a escolha dos sons-alvo, considerando-se a variabilidade e os traços distintivos alterados. Supõe-se que a terapia tornar-se-á mais eficaz. Por outro lado, o Modelo de Oposições Múltiplas ainda não foi testado em crianças falantes do Português Brasileiro (PB).

Desta forma, este relato de caso tem como objetivos propor uma abordagem com enfoque em traços distintivos para o Modelo de Oposições Múltiplas e testar sua aplicação em um sujeito falante do PB.

\section{APRESENTAÇÃO DO CASO CLÍNICO}

\section{Modelo de oposições múltiplas com abordagem em tra- ços distintivos}

Será apresentado o modelo proposto, sendo detalhados os procedimentos para a escolha dos sons-alvo de tratamento, a metodologia terapêutica e a estrutura da sessão recomendada na sua aplicação. Posteriormente, será apresentado o caso clínico em que o modelo foi utilizado.

Este modelo é proposto para DF mais severos e utiliza como técnica o contraste de vários pares de palavras que diferem por apenas um fonema, os quais diferem em vários traços distintivos.

O objetivo dos modelos de oposições é enfatizar a função contrastiva dos fonemas, fazendo com que a criança tente realizar o som-alvo, pois as substituições causam uma quebra na comunicação ${ }^{(8)}$. Com a utilização do contraste de vários fonemas, ao mesmo tempo, a criança percebe a necessidade de se auto corrigir na tentativa de produzir os sons-alvo corretamente e ser compreendida. Neste relato de caso, o Modelo de Oposições Múltiplas com enfoque em traços distintivos tem os mesmos objetivos do modelo original ${ }^{(5)}$, isto é, reorganizar o sistema fonológico intervindo no sistema de sons desviantes, visando a generalização e a melhora na inteligibilidade de fala da criança.

Quanto aos procedimentos para a escolha dos alvos de tratamento, deve-se realizar a coleta e gravação da fala, utilizando técnicas de nomeação e fala espontânea. Sugere-se utilizar um instrumento que contenha figuras de todos os sons do português, nas diferentes posições em que ocorrem. Após a coleta, deve-se realizar a transcrição fonética dos dados, bem como as análises contrastiva e por traços distintivos ${ }^{(9)}$. Pela análise contrastiva, obtém-se o sistema fonológico da criança, sendo possível determinar os fonemas adquiridos, parcialmente adquiridos e os não adquiridos, sendo utilizados os seguintes parâmetros ${ }^{(10)}$ : fonema não adquirido de $0 \%$ a $39 \%$ das possibilidades; parcialmente adquirido de $40 \%$ a $79 \%$; e adquirido de $80 \%$ a $100 \%$. Por intermédio da análise por traços distintivos determinam-se quais os traços distintivos que a criança apresenta dificuldades, sendo importantes para a escolha dos sons-alvos.

Em seguida, os sons-alvo de tratamento são escolhidos com base na análise contrastiva e na de traços distintivos, ou seja, escolher os sons que contenham o conjunto de traços que a criança apresenta dificuldades. Deve-se comparar, dentre os fonemas não adquiridos e parcialmente adquiridos, os traços que estão mais alterados. A seguir, selecionam-se os sons-alvo que devem ser em número superior a três, conter os traços mais alterados, estando todos em oposição múltipla, sendo selecionadas palavras que irão contrastar estes sons sempre na mesma posição na palavra. Essas palavras escolhidas devem ser, preferencialmente, desenháveis, com significado, mas pela dificuldade de se encontrar pares para alguns alvos optou-se por utilizar palavras sem sentido, mas atribuindo conceitos às mesmas, como: apelidos de pessoas, nomes de animais etc.

No que se refere à aplicação terapêutica, deve-se inicialmente realizar a linha de base ${ }^{(3)}$, que é uma forma de sondagem efetuada antes do tratamento fonológico, a fim de obter um parâmetro inicial para comparar os progressos da terapia e as generalizações. Para realizá-la, verificam-se quais são os sons ausentes ou parcialmente adquiridos no sistema fonológico, nas diferentes posições da palavra (onset inicial e medial, coda medial e final, onset complexo). Cada som deve ser testado, selecionando-se seis palavras as quais não apresentem outras dificuldades à exceção do som-alvo, todas diferentes das palavras-alvo e que possam ser representadas por figuras. As figuras devem ser apresentadas à criança, que deve nomeá-las sem o modelo do terapeuta.

Após a determinação da linha de base, inicia-se o tratamento. São realizadas cinco sessões de estimulação com as palavras-alvo e, na sexta, realiza-se a primeira sondagem conforme os procedimentos indicados na linha de base. Continuar o tratamento, realizando sempre cinco sessões e uma sondagem.

O tratamento consiste de duas etapas: imitação e produção. Durante a fase de imitação, a criança deve repetir as palavrasalvo após o modelo dado pelo terapeuta, necessitando atingir um percentual de $80 \%$ ou mais de produções corretas. Assim, na sessão seguinte, deve-se passar para a fase de produção espontânea. Nessa etapa, os alvos devem ser produzidos pela criança sem o modelo imediato do terapeuta. É necessário calcular a porcentagem de produções corretas realizadas pela criança no final de cada sessão. Quando os sons-alvo estiverem adquiridos na sondagem, as palavras utilizadas em terapia devem passar para o nível da sentença, porém alguns alvos podem permanecer no nível da palavra.

Após a realização de 20 a 25 sessões de terapia (não considerar as sessões de sondagem) realizar uma reavaliação do 
sistema fonológico da criança por meio do mesmo instrumento utilizado na avaliação inicial. A partir da coleta e transcrição da fala, realizar a análise contrastiva e por traços distintivos. Os resultados desta reavaliação devem ser comparados com os resultados da linha de base, sondagens, e com a avaliação fonológica inicial, com o propósito de verificar se houve progresso no tratamento. De acordo com as mudanças no sistema fonológico e os resultados da análise por traços distintivos, pode ser necessário ou não selecionar novos alvos ou modelo terapêutico para dar continuidade ao tratamento.

Quanto à estrutura das sessões, estas devem iniciar e terminar com o bombardeio auditivo, o qual consiste em uma lista de quatro a cinco palavras para cada som-alvo, na mesma posição das palavras-alvo, as quais devem ser lidas preferencialmente com amplificação sonora.

A estimulação das palavras-alvo é realizada mediante a prática de produção. Esta consiste em atividades lúdicas como: jogos de memória, boliche, varetas, sinuca, lince, dominó, entre outras, nas quais a criança é estimulada a falar os pares-alvos.

A orientação aos pais ou responsáveis está entre os procedimentos recomendados para a realização de prática de produção em outros ambientes. Os pais ou responsáveis devem ser orientados a ler a lista de palavras do bombardeio auditivo uma vez ao dia para a criança. Além da lista do bombardeio, devem ser entregues, cópias dos desenhos enfatizados em terapia e sugerir atividades para realizarem em casa com a criança.

Se for necessário um trabalho articulatório adicional, exercícios de mobilidade e tonicidade dos órgãos fonoarticulatórios devem ser incluídos na sessão de terapia. Pistas táteis, auditivas e cinestésicas devem auxiliar a criança na imitação e produção isolada dos sons-alvo, bem como auxiliá-la na realização do ponto e modo articulatório do fonema. Esses exercícios, também, devem ser entregues e demonstrados para os pais os realizarem em casa com a criança.

\section{A aplicação do modelo de oposições múltiplas com en- foque em traços distintivos}

O Modelo de Oposições Múltiplas com enfoque em traços distintivos foi aplicado em um sujeito (W.), do sexo masculino, com cinco anos e quatro meses, com DF moderado-severo ${ }^{(11)}$. O menino recebeu atendimento fonoaudiológico em uma clínica escola de uma instituição de ensino superior, estando o projeto devidamente aprovado no Comitê de Ética em Pesquisa (CEP) sob no $108 / 05$.

Primeiramente foram realizadas avaliações fonoaudiológicas (Avaliação da Linguagem, Exame Articulatório, Avaliação do Sistema Estomatognático, Avaliação da Discriminação Auditiva, Avaliação Psicomotora, Avaliação da Consciência Fonológica; Avaliação Simplificada do Processamento Auditivo Central e Avaliação do Vocabulário) e complementares (Avaliação Otorrinolaringológica, Audiológica e Neurológica), a fim de verificar se existiam outras alterações que pudessem excluir o diagnóstico de desvio fonológico. Para a obtenção dos dados da fala utilizou-se a Avaliação Fonológica da Criança $(\mathrm{AFC})^{(9)}$ e, para análise do mesmo, utilizou-se a análise contrastiva e por traços distintivos.

$\mathrm{Na}$ avaliação fonológica inicial de W., em OI (onset inicial) estavam presentes as plosivas /p/, /t/ e /k/, as fricativas /f/, /v/ e /s/, as líquidas /R/ e /l/ e as nasais $/ \mathrm{m} / \mathrm{e} / \mathrm{n} /$. A fricativa $/ \mathrm{S} /$ estava parcialmente adquirida. As plosivas /b/, /d/ e /g/ e a fricativa /3/ estavam ausentes. Em OM (onset medial) as plosivas /p/, /t/ e /k/, a fricativa /f/, as nasais /m/ e /n/ e as líquidas $/ 1 /, / K /$ e /R/ estavam presentes. Estavam parcialmente adquiridos $/ \mathrm{J} / \mathrm{e} / \mathrm{h} /$, e ausentes as plosivas /b/, /d/ e /g/, as fricativas /v/, /s/, /z/, /3/ e a líquida /r/. Em coda medial (CM) o /s/ estava adquirido e $\mathrm{o} / \mathrm{r} /$ ausente. Em coda final (CF), /s/ e /r/ ausentes (Quadro 1).

Considerando-se as substituições que W. realizava na análise fonológica inicial, observou-se dificuldade com os seguintes traços distintivos: [+voz], [-soante], [+coronal/anterior], [-aproximante], [+contínuo] e [labial], sendo que a maior parte das substituições envolvia o traço [+voz].

Para a escolha dos sons-alvo, considerou-se o sistema fonológico inicial (Quadro 1) e os traços em que W. apresentava dificuldades. As maiores dificuldades encontravam-se nos traços: [+voz], [-soante] e [+coronal/-anterior] então, foram selecionados sons que contrastavam esses traços. Dessa forma, foram selecionados os seguintes sons-alvo: /v/ x /s/ x /z/ x /3/ $\mathrm{x} / \mathrm{b} /$ em OI. As palavras-alvo selecionadas para o tratamento fora /vela/ x /scla/ x /zela/ x /3ela/ x /bela/, as quais foram apresentadas, primeiramente, por meio de figuras. Após a realização da linha de base, iniciou-se a terapia propriamente dita. O bombardeio auditivo foi realizado no começo e no final de cada sessão. O bombardeio auditivo e as figuras-alvo

Quadro 1. Avaliação inicial e final do sistema fonológico

\begin{tabular}{|c|c|c|c|c|c|c|}
\hline \multicolumn{7}{|c|}{ Sistema fonológico } \\
\hline \multicolumn{4}{|c|}{ Avaliação inicial } & \multicolumn{3}{|c|}{ Avaliação final } \\
\hline & A & PA & NA & A & PA & NA \\
\hline $\mathrm{OI}$ & $\begin{array}{l}/ \mathrm{p} /, / \mathrm{t} /, / \mathrm{k} /, / \mathrm{f} /, / \mathrm{v} /, / \mathrm{s} / \\
/ \mathrm{R} /, / \mathrm{m} / / \mathrm{n} /, / \mathrm{l} /\end{array}$ & /S/ & $/ \mathrm{b} /, / \mathrm{d} /, / \mathrm{g} /, / 3 /$ & $\begin{array}{l}/ \mathrm{p} /, / \mathrm{t} /, / \mathrm{k} /, / \mathrm{ff} /, / \mathrm{s} /, / \mathrm{J} / \text {, } \\
/ \mathrm{R} /, / \mathrm{m} /, / \mathrm{n} /, / \mathrm{l} /\end{array}$ & & $/ \mathrm{b} /, / \mathrm{d} /, / \mathrm{v} /, / \mathrm{g} /, \mid \mathrm{z} /, / 3 /$ \\
\hline $\mathrm{OM}$ & $\begin{array}{l}/ \mathrm{p} /, / \mathrm{t} /, / \mathrm{k} /, / \mathrm{f} /, / \mathrm{R} /, / \mathrm{m} / \\
/ \mathrm{n} /, / \mathrm{l} /, / \mathrm{N} /\end{array}$ & $/ S /, / \mathrm{h} /$ & $\begin{array}{l}/ \mathrm{b} /, / \mathrm{d} /, / / \mathrm{g} /, / \mathrm{v} /, / \mathrm{s} / \\
\mid \mathrm{z} /, / 3 /, / \mathrm{r} /\end{array}$ & $\begin{array}{l}/ \mathrm{p} /, / \mathrm{t} /, / \mathrm{k} /, / \mathrm{f} /, / \mathrm{s} /, / \mathrm{S} / \\
/ \mathrm{R} /, / \mathrm{m} / / \mathrm{n} /, / \mathrm{l} /, / \mathrm{K} /\end{array}$ & $/ \mathrm{m} /$ & $\begin{array}{l}/ \mathrm{b} /, / \mathrm{d} /, / \mathrm{g} /, / \mathrm{v} /, / \mathrm{z} / \\
/ 3 /, / \mathrm{r} /\end{array}$ \\
\hline $\mathrm{CM}$ & /s/ & & $/ r /$ & /s/ & & $/ \mathrm{r} /$ \\
\hline CF & & & $/ r /$ & /s/ & & $\mid r /$ \\
\hline
\end{tabular}

Legenda: $\mathrm{OI}=$ onset inicial; $\mathrm{OM}=$ onset medial; $\mathrm{CM}=$ coda medial; $\mathrm{CF}=$ coda final; $\mathrm{A}=$ adquirido; $\mathrm{PA}=$ parcialmente adquirido; $\mathrm{NA}=$ não adquirido 
trabalhadas em terapia eram entregues aos pais, que eram orientados para que lessem a lista ao menos uma vez ao dia para a criança e que realizassem atividades com as figuras sugeridas pelo terapeuta. Essa metodologia foi utilizada em outra pesquisa ${ }^{(3)}$, tornando a terapia mais eficaz.

A terapia teve início com a imitação da criança após a produção do terapeuta, ou seja, este falava o nome das figuras e a criança repetia imediatamente. Nas quatro sessões seguintes, a criança tentava produzir as palavras-alvo sem o modelo do terapeuta. Quando a palavra-alvo não era produzida corretamente, a terapeuta trabalhava o som-alvo em nível perceptivo, enfatizando ponto e modo articulatório. Se algumas das palavras não eram lembradas, elas eram novamente nomeadas pela terapeuta não sendo necessário que a criança repetisse imediatamente.

Após dez sessões de terapia, de 45 minutos cada, foi realizada uma nova AFC na qual se observou que os fonemas / $/$ / e /s/ foram adquiridos em OM e / / em OI. O fonema /S/ não era alvo de terapia, no entanto apresentou $100 \%$ de produções corretas na reavaliação. Quanto aos alvos, somente o fonema /s/ foi adquirido (100\%), sendo que os demais sons tratados permaneceram não adquiridos. Pôde-se verificar, também que, dos traços alterados permaneceu somente o [+voz], com exceção do fonema / $/ 3 /$ que permaneceu além do traço [+voz] com o traço [+coronal] (/3/ por [s] e [S]).

\section{DISCUSSÃO}

O modelo aplicado nesta pesquisa enfatizou a estimulação de vários sons-alvo por meio de pares de palavras, sendo que para a seleção destas foram considerados os traços distintivos alterados no sistema fonológico da criança, além do ambiente fonético, pois o único som produzido incorretamente nestas palavras era o som-alvo. Esta proposta não concorda com o pressuposto do Modelo de Oposições Múltiplas ${ }^{(5)}$, pois é preconizado que os sons ausentes/alterados no sistema fonológico devem ser trabalhados em oposição ao fonema utilizado como substituto para todos os sons alterados.

Nessa pesquisa, os traços que estavam alterados eram: [+voz], [-soante], [+coronal/-anterior], [-aproximante], [+contínuo] e [labial], porém os traços que a criança apresentava maiores dificuldades eram: [+voz], [-soante] e [+coronal/ -anterior], justificando a escolha dos seguintes sons-alvo para o tratamento: /v/ x /s/ x /z/ x /3/ x /b/ em OI. Enquanto que em outro estudo, realizado com falantes do inglês ${ }^{(12)}$, a seleção dos sons-alvo foi feita a partir das múltiplas substituições de fonemas por um único som, isto é, uma criança que substitua os fonemas /w/, /y/, /s/, / / por /l/, teria todos esses fonemas, inclusive o fonema substituído, selecionados para tratamento, levando em consideração as possibilidades de ocorrer tais substituições em diferentes sistemas fonológicos, isto é, analisa os traços que podem estar provocando essas trocas.

As palavras utilizadas são preferencialmente com estrutura silábica simples e, quando possível, com sentido conforme as recomendações propostas por diversos autores ${ }^{(2,3,8)}$ para a intervenção nos distúrbios fonológicos. Neste estudo de caso, foram utilizadas duas palavras-alvo de fácil identificação, por se tratarem de substantivos (/vela/x /scla/) e as demais (/zela/ $\mathrm{x} / 3 \varepsilon \mathrm{la} / \mathrm{x} / \mathrm{b} \varepsilon \mathrm{la} /)$ foram previamente conceituadas junto ao paciente, sendo explicado o contexto da figura já que se trata de verbos e adjetivo. Pela grande variedade de sons-alvo escolhidos nesse modelo e por não se ter no Português Brasileiro tantas possibilidades de pares de palavras de fácil identificação e contraste, como na língua inglesa, esse recurso foi utilizado. Outro estudo ${ }^{(7)}$ também mostrou que nem sempre é possível encontrar palavras com significados reais para a terapia de oposições múltiplas, lançando mão das palavras sem sentido com intuito de manter a estrutura da palavra.

O Modelo de Oposições Múltiplas com enfoque em traços distintivos foi eficaz no tratamento deste sujeito. Verificaram-se melhoras no sistema fonológico após o tratamento. Apesar de poucas sessões de terapia, o sujeito generalizou para outros fonemas e outras posições na palavra, por exemplo: o /s/, tratado em OI, foi generalizado para OM, e houve melhoras no / / em OI e OM o qual não foi estimulado em terapia. A interação entre a avaliação, seleção do alvo e intervenção podem resultar em menor tempo de tratamento Williams ${ }^{(7,13)}$. O objetivo do tratamento é conseguir a reorganização fonológica máxima com o menor tempo de intervenção ${ }^{(12)}$.

A proposta deste modelo tem como finalidade abranger as maiores dificuldades da criança em termos de traços distintivos para assim contrastá-los em terapia. A partir disso, espera-se que no decorrer do tratamento, esse sujeito venha a adquirir os sons tratados, bem como, apresente generalizações para outras palavras, outras posições na palavra e para outras classes de sons. Pesquisas têm revelado que crianças adquirem mais rapidamente os fonemas, quando o tratamento tem como objetivo a generalização, ao invés de ensinar um fonema de cada vez e em cada posição ${ }^{(8,13)}$.

\section{COMENTÁRIOS FINAIS}

Com este relato de caso, que teve como objetivos propor uma abordagem com enfoque em traços distintivos para o Modelo de Oposições Múltiplas e testar sua aplicação em um sujeito falante do Português Brasileiro, concluiu-se que:

- a aplicação do modelo proposto foi baseada em procedimentos recomendados no tratamento com base fonológica, sendo que muitos desses procedimentos também são utilizados em outros modelos de terapia;

- o modelo de oposições múltiplas com enfoque em traços distintivos mostrou-se efetivo na aplicação de sujeitos com desvios fonológicos compatíveis com a proposta, pois houve melhoras consideráveis no sistema fonológico, mesmo com um período curto de terapia;

- para a escolha dos alvos de terapia deve-se considerar o sistema fonológico inicial, assim como os traços distintivos alterados com maior frequência. Isso favoreceu as mudanças fonológicas obtidas por meio do contraste dos traços distintivos mais alterados, favorecendo a generalização. 


\begin{abstract}
The aim of this study was to propose an approach based on distinctive features for the Multiple Oppositions Model, and to test its application on a Brazilian Portuguese speaker. The model was used with a male subject with moderate-severe phonological disorder and six years and six months old. After phonological evaluation the target sounds were selected. The initial phonological system was considered, as well as the altered distinctive features ([+voiced], [+sonant], [+approximant], [coronal/_tanterior], [-continuous]). The features with more alterations were [+voiced, +sonant, +approximant], justifying the choice of the following target sounds for treatment: $/ \mathrm{r} / \mathrm{x} / \mathrm{l} / \mathrm{x} / \mathrm{K} / \mathrm{x} / 3 / \mathrm{x} / \mathrm{z} /$, in medial onset. Based on the application of this model in English speakers, some necessary procedures were introduced, such as: choice of the target-sounds based on the altered distinctive features, and the structure of therapy session. At the end, the subject was reassessed, in order to verify the phonological system acquisitions. After ten speech-language therapy sessions, the subject acquired the phonemes $/ 1, \kappa, 3, \mathrm{z} /$, generalizing them to other word positions (/l/ and $/ 3 /$ in initial onset). It was also verified the occurrence of generalization for the same class and other classes of sounds $(/ \mathrm{b} /, / \mathrm{d} /, / \mathrm{g} /, / \mathrm{v} /)$. However, the phoneme /r/ was not acquired. This proposal proved to be effective, for the subject showed improvement on his phonological system with few therapeutic sessions. It was possible to observe that the Multiple Oppositions Model, when applied using the approach based on distinctive features, is effective, because it favored many phonological changes.
\end{abstract}

Keywords: Speech disorders; Speech; Speech therapy; Generalization; Children; Case reports

\section{REFERÊNCIAS}

1. Tyler AA, Edwards ML, Saxman JH. Clinical application of two phonologically based treatment procedures. J Speech Hear Disord. 1987;52(4):393-409.

2. Gierut JA. Maximal opposition approach to phonological treatment. J Speech Hear Disord. 1989;54(1):9-19.

3. Bagetti T, Mota HB, Keske-Soares M. Modelo de oposições máximas modificado: uma proposta de tratamento para o desvio fonológico. Rev Soc Bras Fonoaudiol. 2005;10(1):36-42.

4. Tyler AA, Figurski GR. Phonetic inventory changes after treating distinctions along an implicational hierarchy. Clin Linguist Phon. 1994;8(2):91-107.

5. Williams AL. Multiple oppositions: theoretical foundations for an alternative contrastive intervention approach. Am J Speech Lang Pathol. 2000;9(4):282-8.

6. Williams L. On "Minimal Pair Approaches to Phonological Remediation,” (Semin speech lang 2002;23:57-68). Semin Speech Lang. 2003;24(3):257-8.
7. Williams AL. A systematic perspective for assessment and intervention: a case study. Int J Speech Lang Pathol. 2006;8(3):245-56.

8. Pereira LF, Mota HB. Tratamento fonológico baseado nos contrastes de oposições máximas. Pró-fono. 2002;14(2):165-74.

9. Yavas MS, Hernandorena CLM, Lamprecht RR. Avaliação fonológica da criança: reeducação e terapia. Porto Alegre: Artes Médicas; 1992. 148p.

10. Bernhardt B. Developmental implications of nonlinear phonological theory. Clin Linguist Phon. 1992;6(4):259-81.

11. Shriberg LD, Kwiatkowski J. Phonological disorders I: a diagnostic classification system. J Speech Hear Disord. 1982;47(3):226-41.

12. Williams AL. Multiple oppositions: case studies of variables in phonological intervention. Am J Speech Lang Pathol. 2000;9(4):289-99.

13. Williams AL. Assessment, target selection, and intervention: dynamic interactions within a systemic perspective. Top Lang Disord. 2005;25(3):231-42. 